\title{
BEAM-BEAM INSTABILITY DRIVEN BY WAKEFIELD EFFECTS IN LINEAR COLLIDERS
}

\author{
R. Brinkmann, DESY, Hamburg, Germany \\ O. Napoly, CEA/Saclay, Gif-sur-Yvette, France \\ D. Schulte, CERN, Geneva, Switzerland
}

\begin{abstract}
The vertical beam profile distortions induced by wakefield effects in linear colliders (the so-called "banana effect") generate a beam-beam instability at the collision point when the vertical disruption parameter is large. We illustrate this effect in the case of the TESLA linear collider project. We specify the tolerance on the associated emittance growth, which translates into tolerances on injection jitter and, for a given tuning procedure, on structure misalignments. We look for possible cures based on fast orbit correction at the interaction point and using a fast luminosity monitor.
\end{abstract}

\section{INTRODUCTION}

In order to obtain the highest luminosity, the beam parameters at the interaction point (IP) of high-energy linear $\mathrm{e}^{+} \mathrm{e}^{-}$colliders, are pushed to the limit of tolerable beamstrahlung and beam-beam background. Consequently, the beam-beam interaction is very intense even for flat beams with large aspect ratio $R=\sigma_{x}^{*} / \sigma_{y}^{*}$. Accordingly the vertical disruption parameter $D_{y}$, defined as the ratio of the bunch length $\sigma_{z}$ to the vertical beam-beam focal length $f_{y}$, and given by

$$
D_{y} \equiv \frac{\sigma_{z}}{f_{y}}=\frac{2 r_{e} N_{b} \sigma_{z}}{\gamma \sigma_{x}^{*}\left(\sigma_{x}^{*}+\sigma_{y}^{*}\right)}
$$

is much larger than one. Table 1 lists the IP design parameters for the TESLA [1]. The sensitivity of the luminosity to offset and angle errors is shown in Fig.1: it is enhanced by a factor 5 for small offsets and by a factor 10 for small angles with respect to the simple geometric factor. This optimisation of the IP parameters into a high disruptive regime is justified by the ability of the fast inter-bunch orbit feed-

Table 1: TESLA $500 \mathrm{GeV}$ parameters

\begin{tabular}{|c|c|c|}
\hline Center of mass energy $[\mathrm{TeV}]$ & $2 E_{0}$ & 0.5 \\
\hline Luminosity $\quad\left[10^{34} \mathrm{~cm}^{-2} \mathrm{~s}^{-1}\right]$ & $\mathcal{L}$ & 3.4 \\
\hline Bunch population & $N_{b}$ & $2 \times 10^{10}$ \\
\hline Spot sizes & $\sigma_{x}^{*}, \sigma_{y}^{*}$ & 553,5 \\
\hline Bunch divergences & $\Theta_{x}^{*}, \Theta_{y}^{*}$ & 37,12 \\
\hline Norm. emittances [mm.mrad] & $\gamma \epsilon_{x}, \gamma \epsilon_{y}$ & $10,0.030$ \\
\hline Beta functions & $\beta_{x}^{*}, \beta_{y}^{*}$ & $15,0.4$ \\
\hline Bunch length & $\sigma_{z}$ & 300 \\
\hline Disruption parameters & $D_{x}, D_{y}$ & $0.22,25$ \\
\hline
\end{tabular}

back to maintain the beams in collision to the specified tolerances.

In this paper the luminosity loss due to internal bunch deformations is investigated. The single-bunch wakefields in the linacs lead to a vertical offset and angle $\left(y_{S}(z), y_{S}^{\prime}(z)\right)$ of the slice centres as a function of their longitudinal position $z$ in the bunch. For large disruption, this can cause a collective kink instability [2] which reduces the luminosity.

The TESLA $500 \mathrm{GeV}$ collider parameters are considered, where the expected emittance growth and wakefield distorsions from the linac are small but the vertical disruption is very large.

\section{SIMULATION PROCEDURE}

Assuming that the Gaussian beam size and divergence at the IP are not affected by the linac transport and are not correlated to the $z$-position, the 3-d correlated distribution function $\rho\left(y, y^{\prime}, z\right)$ of the bunch at the IP is given by

$$
\begin{aligned}
\rho\left(y, y^{\prime}, z\right)= & \frac{1}{2 \pi \sigma_{y}^{*} \Theta_{y}^{*}} \rho(z) \\
& \exp \left(-\frac{\left(y-y_{S}(z)\right)^{2}}{2 \sigma_{y}^{* 2}}-\frac{\left(y^{\prime}-y_{S}^{\prime}(z)\right)^{2}}{2 \Theta_{y}^{* 2}}\right)
\end{aligned}
$$

where $\rho(z)$ is the normalized longitudinal distribution. The amplitude of these deformations can be quantified by the vertical emittance $\epsilon_{y}$ given by

$$
\begin{aligned}
\epsilon_{y}^{2}= & \left(\sigma_{y}^{* 2}+\int d z \rho(z)\left(y_{S}(z)-\left\langle y_{S}\right\rangle\right)^{2}\right) \times \\
& \left(\Theta_{y}^{* 2}+\int d z \rho(z)\left(y_{S}^{\prime}(z)-\left\langle y_{S}^{\prime}\right\rangle\right)^{2}\right)
\end{aligned}
$$

where $\left\langle y_{S}\right\rangle=\int d z \rho(z) y_{S}(z)$ and $\left\langle y_{S}^{\prime}\right\rangle=\int d z \rho(z) y_{S}^{\prime}(z)$. The impact of the bunch deformation $\left(y_{S}, y_{S}^{\prime}\right)$ on the emittance growth is obvious from this equation. Colliding $\mathrm{e}^{+}$ with $\mathrm{e}^{-}$bunches with different deformations the resulting luminosity is defined by the double sum which integrates the $e^{+}$and $e^{-}$slice overlaps over all longitudinal positions. To separated the simple geometric decay of the luminosity due to the 'banana' shape of the bunches, from the effect of the beam-beam instability, it is useful to introduce both the luminosity $\mathcal{L}_{0}$ calculated by propagating the longitudinal slices without beam-beam forces, and the luminosity $\mathcal{L}$ with beam-beam forces in action.

Two main effects contribute to the correlated emittance growth in the TESLA linac: a beam offset in the structures, because of the transverse wakefield $W_{\perp}(z)$, and a 

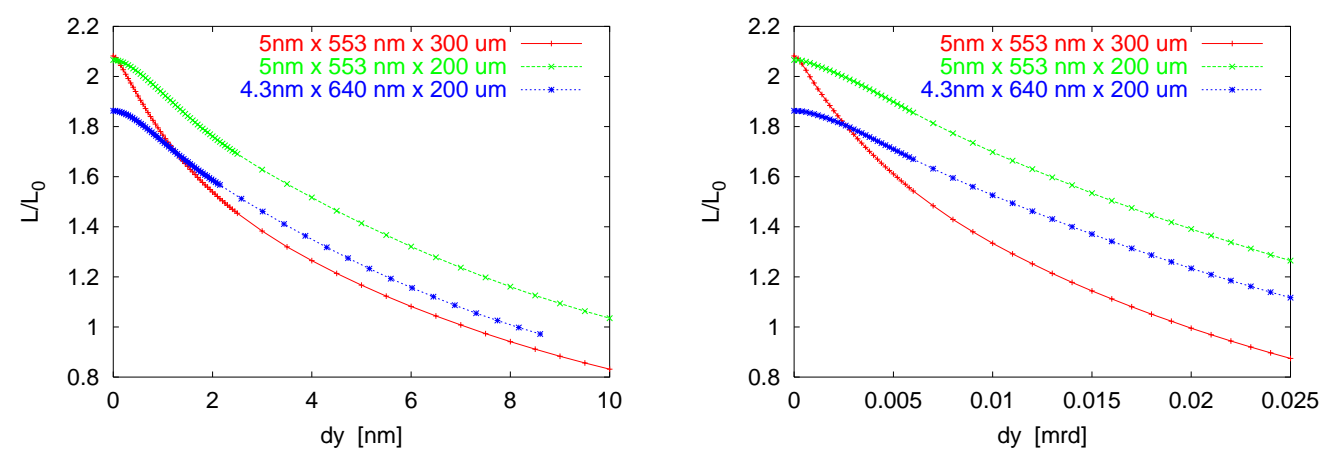

Figure 1: Comparison of the decay of the luminosity with vertical IP offset (left) and vertical IP angle (right) for the nominal TESLA parameters $\sigma_{z}=300 \mu \mathrm{m}$ and two other sets of parameters with a reduced bunch length $\sigma_{z}=200 \mu \mathrm{m}$ and a smaller vertical disruption parameter.

beam offset in the quadrupoles, because of the beam energy spread induced by the longitudinal wake field $W_{\|}(z)$. Both effects can originate from static (i.e. stable over a large number of pulses) misalignments and from jittery (i.e. varying from pulse to pulse) linac injection errors. For the TESLA assumptions concerning misalignments $(500 \mu \mathrm{m}$ RMS for cavities, $300 \mu \mathrm{m}$ RMS for quadrupoles and 1$\sigma$ injection errors) the expected emittance growth [6] are in the order or below $10 \%$, depending on the beam-based linac alignment methods. In this perturbative regime, the bunch deformations $\left(y_{S}, y_{S}^{\prime}\right)$ can be described to first order by linear combinations of the wake potentials themselves:

$$
\begin{aligned}
& y_{S}(z)=A W_{\|}(z)+B W_{\perp}(z) \\
& y_{S}^{\prime}(z)=A^{\prime} W_{\|}(z)+B^{\prime} W_{\perp}(z)
\end{aligned}
$$

As in Eq.1, the transverse beam matrix of the slices is constant along the bunch with an emittance slightly degraded with respect to the damping ring emittance. For our simulations, we will therefore compare the case where the slice emittance is equal to damping ring (DR) design emittance $\gamma \epsilon_{y, 0}=0.02 \mu \mathrm{m}$ to the case where it is equal to the design IP emittance $\gamma \epsilon_{y, 0}=0.03 \mu \mathrm{m}$ (i.e. allowing for $50 \%$ dilution). For the design IP optics $\left(\beta_{y}^{*}=400 \mu \mathrm{m}\right)$ the beam matrix is upright and the vertical spot size of the slices is $5 \mathrm{~nm}$ for the latter case, and $4.1 \mathrm{~nm}$ for the first one.

The following results have been obtained by simulating the beam in the TESLA linac with PLACET[4] and the beam-beam interaction with GUINEA-PIG[3]. An average over 100 cases is always calculated. Some of the results have been cross checked by using the analytical bunch deformations in Eqs.3 and calculating the luminosity with the beam-beam programme BBSIM [5] which fits the slice transverse distributions by a double Gaussian and calculates the beam-beam potentials analytically. Both methods gave comparable results.

\section{RESULTS}

The effect of beam jitter at injection is simulated first. The average beam angle and position is assumed to be corrected to zero by a feedback at the end of the linac. Figure 2 shows the effect of injection jitter with an RMS of up to 3$\sigma$ in both $y$ and $y^{\prime}$. Note that no optimisation of the vertical waist position is performed which explains the $15 \%$ lower luminosity for zero jitter with respect to the parameter list in Table 1 . The average emittance growth is about $11 \%$ for the 3- $\sigma$ jitter and scales roughly quadratically with the jitter amplitude. In all cases the luminosity loss $\Delta \mathcal{L}$ is 5 to 30 times larger than calculated for the geometric luminosity $\mathcal{L}_{0}$, which itself roughly scales as the square root of the inverse of the vertical emittance given by Eq.2.

Figure 2 also shows the luminosity for shorter bunches. For simplification and to only show the beam-beam effects, the bunches have the same shape, simply scaled down in length. In this case the sensitivity of the luminosity is strongly decreased. This is explained by the smaller disruption parameter $D_{y}$ which, as shown by Fig.1, reduces the beam-beam instability. For the same reason, the luminosity is more sensitive for a smaller vertical emittance and IP spot size, since the disruption parameter increases. However, for the design DR emittance $\gamma \epsilon_{y, 0}=0.02 \mu \mathrm{m}$ and the design bunch length $\sigma_{z}=300 \mu \mathrm{m}$ the luminosity loss for 1- $\sigma$ injection jitter is compensated by the smaller spot size and higher luminosity per slices in such a way that the design TESLA luminosity is practically recovered.

Static misalignments are simulated next. They result in an emittance growth of about $10 \%$ on average. For the design IP emittance, the average luminosity is $70 \%$ of the design value. For the DR emittance it is $75 \%$.

However, most of the luminosity can be restored by some optimisation of the collision parameters which could take place within the bunch train. The fastest optimization concerns the relative beam offset and angle. An example is shown in Fig.3(top) where a typical collision of bunches with $\gamma \epsilon_{y, 0}=0.03 \mu \mathrm{m}$ and $\sigma_{z}=300 \mu \mathrm{m}$ is considered where the luminosity is about $70 \%$ lower than design for zero relative offset and angle. An offset scan followed by an angle scan re-optimize the beam relative offset and angle to setpoints where the luminosity drops only by less than $10 \%$. In practice, more scans could follow these two initial ones. One could also implement fast quadrupole scans to 


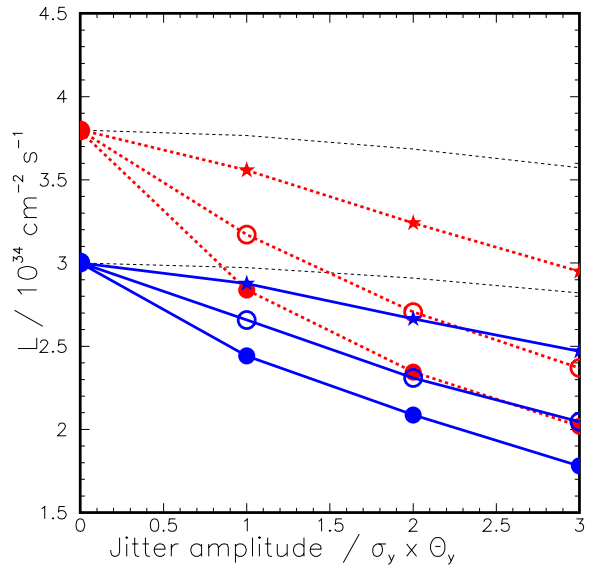

Figure 2: Luminosity as a function of the RMS linac injection jitter for the nominal IP emittance $\gamma \epsilon_{y, 0}=0.03 \mu \mathrm{m}$ (solid) and for the nominal DR emittance $\gamma \epsilon_{y, 0}=0.02 \mu \mathrm{m}$ (dashed) in the slices. The luminosity $\mathcal{L}$ calculated for $\sigma_{z}=300 \mu \mathrm{m}$ (full circle), $\sigma_{z}=200 \mu \mathrm{m}$ (empty circle) and $\sigma_{z}=100 \mu \mathrm{m}$ (star) is compared to the geometric luminosity $\mathcal{L}_{0}$ (thin line).

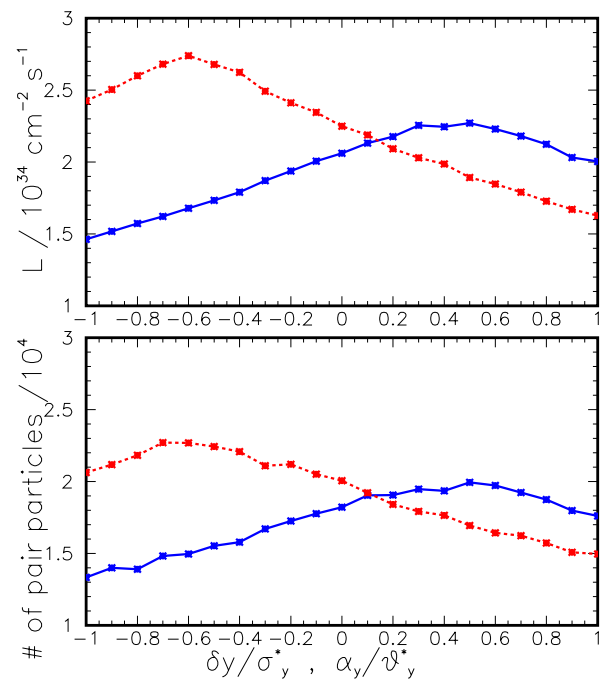

Figure 3: Optimisation the luminosity for a typical linac static misalignment by scanning the average beam offset $\delta y^{*}$ (solid) and then the relative beam angle $\alpha_{y}^{*}$ (dashed). The middle plot shows the response of the pair monitor, and the bottom plot the beam-beam kick

optimize the vertical phase space disributions of one bunch relative to the other. Such scans rely onthe ability to actually monitor the luminosity. The $\mathrm{e}^{+} \mathrm{e}^{-}$pairs signal can be used [7] for that purpose, as shown by Fig.3(bottom).

As another consequence of the instability, the beambeam kick will be non-zero even for zero relative collision offset. Also the dependence of the kick angle on the offset is modified. This will affect the intra-pulse interaction-

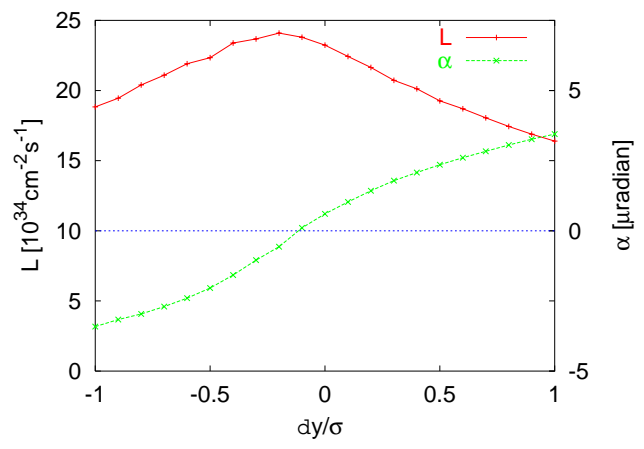

Figure 4: Optimisation the luminosity for a typical beam jitter in the linac. The feedback minimises the angle kick, recovering some luminosity but not finding the optimum offset.

point feedback, which relies on the BPM measurements of the beam-beam kick. It will let the beams collide with an offset, to achieve zero kick angle. Simulations show that this effect is on average benificial as it recovers a fraction of the luminosity loss. Figure 4 illustrates with an example.

\section{CONCLUSION}

In the linac of TESLA, wakefield effects lead to vertical distortions of the transported beam. Conventionally, these distortions are summarised as an emittance growth $\Delta \epsilon_{y}$. In the presence of strong beam-beam effects this emittance growth is not a good measure of the luminosity loss $\Delta \mathcal{L}$. A very small growth of $\Delta \epsilon_{y} / \epsilon_{y}=1.3 \%$ can lead to $\Delta \mathcal{L} / \mathcal{L}=$ $25 \%$ rather than the expected $\Delta \mathcal{L} / \mathcal{L}=0.7 \%$.

By adjusting the offset and crossing angle of the two beams, the luminosity loss due to static misalignments can be reduced significantly. The necessary measurement of the luminosity can be provided by monitoring the total number of $\mathrm{e}^{+} \mathrm{e}^{-}$pairs produced.

\section{REFERENCES}

[1] R. Brinkmann, K. Flöttmann, J. Roßbach, P. Schmüser, N. Walker and H. Weise (eds.), "TESLA Technical Design Report, Part II The Accelerator", DESY 2001-011, http://tesla.desy.de/new_pages/TDR_CD/PartII/accel.html

[2] Y.H. Chin, DESY preprint DESY-87-011.

[3] D. Schulte, Beam-Beam Simulation with GUINEA-PIG. Proc. of ICAP 1998, Monterey, USA (1998).

[4] E. D'Amico, N. Leros, G. Guignard and D. Schulte. This conference.

[5] R. Brinkmann, presented at the LC92 Int. Linear Collider Workshop, Garmisch-Patenkirchen Aug. 1992, unpublished.

[6] G.A. Amutuni et al, "On the Single Bunch Emittance Preservation in TESLA”, DESY-TESLA Note 2001-02

[7] "Luminosity Monitor Options for TESLA", D. Schulte and O. Napoly, Proc. of the XIX International LINAC Conference, Chicago, USA (1998). 